\title{
A Discrete-Time Queue with Preferred Customers and Partial Buffer Sharing
}

\author{
Shizhong Zhou, ${ }^{1,2}$ Liwei Liu, ${ }^{1}$ and Jianjun $\mathrm{Li}^{3}$ \\ ${ }^{1}$ Department of Statistics and Financial Mathematics, School of Science, Nanjing University of Science and Technology, \\ Nanjing, Jiangsu 210094, China \\ ${ }^{2}$ School of Science, Guangxi University for Nationalities, Nanning, Guangxi 530006, China \\ ${ }^{3}$ Nanjing University of Science and Technology, Nanjing, China
}

Correspondence should be addressed to Shizhong Zhou; zhoushizhong666@sina.com

Received 16 April 2015; Revised 26 June 2015; Accepted 7 July 2015

Academic Editor: Francesco Soldovieri

Copyright (C) 2015 Shizhong Zhou et al. This is an open access article distributed under the Creative Commons Attribution License, which permits unrestricted use, distribution, and reproduction in any medium, provided the original work is properly cited.

\begin{abstract}
We analyze a discrete-time Geo/Geo/1 queueing system with preferred customers and partial buffer sharing. In this model, customers arrive according to geometrical arrival processes with probability $\lambda$. If an arriving customer finds the server idle, he begins instantly his services. Otherwise, if the server is busy at the arrival epoch, the arrival either interrupts the customer being served to commence his own service with probability $\theta$ (the customer is called the preferred customer) or joins the waiting line at the back of the queue with probability $\widetilde{\theta}$ (the customer is called the normal customer) if permitted. The interrupted customer joins the waiting line at the head of the queue. If the total number of customers in the system is equal to or more than threshold $N$, the normal customer will be ignored to enter into the system. But this restriction is not suitable for the preferred customers; that is, this system never loses preferred customers. A necessary and sufficient condition for the system to be stable is investigated and the stationary distribution of the queue length of the system is also obtained. Further, we develop a novel method to solve the probability generating function of the busy period of the system. The distribution of sojourn time of a customer in the server and the other indexes are acquired as well.
\end{abstract}

\section{Introduction}

The queuing system in which the interarrivals and service times are positive integer values of random variables is called the discrete-time queuing system. Compared with the continuous time, the discrete-time queue is more suitable for the modeling and performance analysis of computer systems, telecommunication network systems, manufacturing and production systems, traffic systems, and health-care systems. Moreover, the discrete-time modeling can be used to approximate the continuous system in practice, but the reverse is generally not true [1]. Since the advent of Meisling's creative paper [2] on the discrete-time queuing in 1958, theoretical research in this filed has become a highlighted topic and the range of applications extends to a number of areas in real life. For a detailed discussion and applications of the discrete-time queueing system one can be referred to the books $[1,3,4]$ and the references therein.
In the last 20 years a number of the discrete-time queueing models have been studied, details of which may be found in papers [5-7]. The preemptive queuing also became the focus of discussion by the papers such as [8-10]. Particularly in [11], a novel recursive method for Geol/Geo2/G/1/K+1 queue with partial buffer sharing was developed. In 2010, Jolai et al. [8] considered a preemptive discrete-time priority buffer system with partial buffer sharing. In that paper, they obtained the steady probability of the system by using the recursive method. Atencia and Pechinkin [12] studied the discrete-time queueing system under the optional LCFS discipline in 2013. Wu et al. [13] analyzed the discrete-time retrial queueing system with preferred and impatient customers in 2013 .

The intention of the present paper is to study the $\mathrm{Geo} / \mathrm{Geo} / 1$ queueing system with preferred customers and partial buffer sharing. As everyone knows, the analysis of a queue system with partial buffer sharing is much more 
difficult than that with infinite capacity. In the partial buffer sharing mechanism, the normal arrivals can only access the buffer if the buffer occupancy is less than a predetermined value $N$. In order to utilize the capacity of the space priority queue more for high-priority customers (or the preferred customers), the threshold $N$ has been chosen to restrict the entrance of the low-priority customers (or the normal customers).

The remaining sections of this paper are organized as follows: in the next section, the model description and assumptions are stated. In Section 3, the one-step transition probabilities and the equilibrium equations are determined. In Section 4, a necessary and sufficient condition for the system to be stable is investigated and the steady-state probability distribution of the system is calculated. In Section 5, the performance measures of the system are provided such as the loss probability, the average number of customers, the probability generating functions of the busy period of the system, and the sojourn time of a customer in a server. The numerical results are discussed in Section 6. The last section is the conclusion.

\section{Model Description and Assumptions}

We consider a Geo/Geo/1 queueing system with preferred customers and partial buffer sharing. In this system, the server may start his service only at discrete-time dots. Thus the service time axis can be decomposed into an integer number of time slots. Without loss of generality, we assume that it is the unit service time slots. It is supposed that all queueing activities of arriving and departing happen at the slot boundaries. That is, they may take place at the same time. But, for convenience, we assume, like the disposed method by many papers concerning the discrete-time queue, that a potential arrival occurs in the interval $\left(m^{-}, m\right)$ and a potential departure takes place in the interval $\left(m, m^{+}\right)$. That is, the arrivals occur at the epoch just before the slot boundaries and the departures occur at the epoch just after the slot boundaries.

We assume that the arrival process is Bernoulli process and the intertime intervals of the arrivals follow the same geometric distribution with rate $\lambda$. The service times are independent and identically distributed according to a geometric distribution with $\mu$. Further, we suppose that the arrival process and the service process are mutually independent.

If an arriving customer finds the server idle, he commences immediately their services. Otherwise, if the server is busy at the arrival epoch, the arrival either interrupts the customer being served to commence his own service with probability $\theta$ or joins the waiting line at the back of the queue with probability $\tilde{\theta}$ if permitted. The interrupted customer joins the waiting line at the head of the queue.

The queueing system has a threshold for buffer control. If the total number of customers in the system is equal to or more than threshold $N$, the normal will be discarded to join the waiting line by the system. But this limitation is not suitable for the preferred customer. That is, the preferred arrival could always enter into the system and interrupts the customers being served no matter how long the queue length is. It is observed that, for $\theta=0$, the model is the standard $\mathrm{Geo} / \mathrm{Geo} / 1 / N$ queueing system in the late arrivals at observed epoch $m^{+}$, while, for $\theta=1$, the model is the standard $\mathrm{Geo} / \mathrm{Geo} / 1 / \infty$ queueing system with interrupted service under the LCFS discipline in the late arrivals at observed epoch $m^{+}$.

\section{One-Step Transition Probabilities and Equilibrium Equations}

The system can be described by the process $\left\{Y_{m}, m=\right.$ $0,1,2, \ldots\}$, where $Y_{m}$ represents the number of the customers in the system at epoch $m^{+}$. Obviously, $\left\{Y_{m}, m=0,1,2, \ldots\right\}$ is a Markov chain. Let $\pi_{k}$ be the stationary probability, at the epoch noted by $m^{+}$, that there are $k$ customers in the system. By the description above it is easy to see that the system obeys the following equilibrium equations:

$$
\begin{aligned}
& \pi_{0}=\tilde{\lambda} \mu \pi_{1}+\tilde{\lambda} \pi_{0}, \\
& \pi_{1}=\tilde{\lambda} \mu \pi_{2}+\lambda \widetilde{\theta} \mu \pi_{1}+\tilde{\lambda} \tilde{\mu} \pi_{1}+\lambda \pi_{0}, \\
& \pi_{k}=\tilde{\lambda} \mu \pi_{k+1}+\lambda \widetilde{\theta} \mu \pi_{k}+\tilde{\lambda} \tilde{\mu} \pi_{k}+\lambda \theta \pi_{k-1}+\lambda \tilde{\theta} \widetilde{\mu} \pi_{k-1}, \\
& 2 \leq k \leq N-2 \text {, } \\
& \pi_{N-1}=\tilde{\lambda} \mu \pi_{N}+\lambda \widetilde{\theta} \mu \pi_{N}+\lambda \widetilde{\theta} \mu \pi_{N-1}+\tilde{\lambda} \tilde{\mu} \pi_{N-1} \\
& +\lambda \theta \pi_{N-2}+\lambda \tilde{\theta} \tilde{\mu} \pi_{N-2}, \\
& \pi_{N}=\tilde{\lambda} \mu \pi_{N+1}+\lambda \tilde{\theta} \mu \pi_{N+1}+\lambda \tilde{\theta} \tilde{\mu} \pi_{N}+\tilde{\lambda} \tilde{\mu} \pi_{N} \\
& +\lambda \theta \pi_{N-1}+\lambda \tilde{\theta} \tilde{\mu} \pi_{N-1}, \\
& \pi_{k}=\tilde{\lambda} \mu \pi_{k+1}+\lambda \widetilde{\theta} \mu \pi_{k+1}+\lambda \tilde{\theta} \widetilde{\mu} \pi_{k}+\tilde{\lambda} \widetilde{\mu} \pi_{k}+\lambda \theta \pi_{k-1}, \\
& k \geq N+1 \text {. }
\end{aligned}
$$

The normalization condition is

$$
\sum_{k=0}^{\infty} \pi_{k}=1 .
$$

\section{Analysis of the System}

In this section we will analyze the discrete-time Geo/Geo/1 queueing system with preferred customers and partial buffer sharing.

Using a recursive method, we can calculate the steadystate probability distribution of the system. From (1) and (2), we obtain

$$
\begin{aligned}
& \pi_{1}=\frac{\lambda}{\widetilde{\lambda} \mu} \pi_{0}, \\
& \pi_{2}=\frac{\lambda^{2}}{(\widetilde{\lambda} \mu)^{2}}(1-\widetilde{\theta} \mu) \pi_{0} .
\end{aligned}
$$

According to (3), we have

$$
(\tilde{\lambda} \mu+\lambda \theta+\lambda \tilde{\theta} \widetilde{\mu}) \pi_{k}=\tilde{\lambda} \mu \pi_{k+1}+(\lambda \theta+\lambda \tilde{\theta} \widetilde{\mu}) \pi_{k-1}
$$


or equivalently

$$
\pi_{k+1}-\pi_{k}=\frac{\lambda}{\tilde{\lambda} \mu}(1-\tilde{\theta} \mu)\left(\pi_{k}-\pi_{k-1}\right) .
$$

Hence

$$
\begin{aligned}
\pi_{k+1}= & \left(\pi_{k+1}-\pi_{k}\right)+\left(\pi_{k}-\pi_{k-1}\right)+\cdots+\left(\pi_{2}-\pi_{1}\right) \\
& +\pi_{1} \\
= & {\left[\frac{\lambda}{\widetilde{\lambda} \mu}(1-\tilde{\theta} \mu)\right]^{k-1}\left(\pi_{2}-\pi_{1}\right) } \\
& +\left[\frac{\lambda}{\widetilde{\lambda} \mu}(1-\tilde{\theta} \mu)\right]^{k-2}\left(\pi_{2}-\pi_{1}\right)+\cdots \\
& +\left(\pi_{2}-\pi_{1}\right)+\pi_{1}=\frac{\lambda}{\tilde{\lambda}_{\mu}}\left(\alpha^{*}\right)^{k} \pi_{0},
\end{aligned}
$$

where $\alpha^{*}=\lambda(1-\tilde{\theta} \mu) / \widetilde{\lambda} \mu$.

That is,

$$
\pi_{k}=\frac{\lambda}{\tilde{\lambda}_{\mu}}\left(\alpha^{*}\right)^{k-1} \pi_{0}, \quad 1 \leq k \leq N-1 .
$$

From (4) and (12), we get

$$
\pi_{N}=\frac{\alpha}{\theta}\left(\alpha^{*}\right)^{N-1} \pi_{0},
$$

where $\alpha=\lambda \theta / \mu(1-\lambda \theta)$.

Also by (5), (12), and (13), we have

$$
\pi_{N+1}=\alpha^{2}\left(\alpha^{*}\right)^{N-1} \pi_{0} .
$$

We can rewrite (6) as

$$
(\lambda \theta+\tilde{\lambda} \mu+\lambda \widetilde{\theta} \mu) \pi_{k}=(\widetilde{\lambda} \mu+\lambda \widetilde{\theta} \mu) \pi_{k+1}+\lambda \theta \pi_{k}
$$

or

$$
\pi_{k+1}-\pi_{k}=\alpha\left(\pi_{k}-\pi_{k-1}\right) .
$$

Therefore, we have

$$
\begin{aligned}
\pi_{k+1}= & \left(\pi_{k+1}-\pi_{k}\right)+\left(\pi_{k}-\pi_{k-1}\right)+\cdots+\left(\pi_{N+1}-\pi_{N}\right) \\
& +\pi_{N} \\
= & (\alpha)^{k-N}\left(\pi_{N+1}-\pi_{N}\right)+(\alpha)^{k-N-1}\left(\pi_{N+1}-\pi_{N}\right) \\
& +\cdots+\left(\pi_{N+1}-\pi_{N}\right)+\pi_{N} \\
= & \frac{1-(\alpha)^{k-N+1}}{1-\alpha}\left(\pi_{N+1}-\pi_{N}\right)+\pi_{N} \\
= & \frac{1}{\theta}\left(\alpha^{*}\right)^{N-1}(\alpha)^{k-N+2} \pi_{0}, \quad k \geq N+1 .
\end{aligned}
$$

Note that (17) is also correct for $k=N$.
We finally obtain

$$
\pi_{k}= \begin{cases}\frac{\lambda}{\widetilde{\lambda} \mu}\left(\alpha^{*}\right)^{k-1} \pi_{0}, & 1 \leq k \leq N-1, \\ \frac{1}{\theta}\left(\alpha^{*}\right)^{N-1}(\alpha)^{k-N+1} \pi_{0}, & k \geq N .\end{cases}
$$

The normalization condition can be written as

$$
\pi_{0}+\sum_{k=1}^{N-1} \pi_{k}+\sum_{k=N}^{\infty} \pi_{k}=1 .
$$

Thus, we can find the unknown constant $\pi_{0}$; that is,

$$
\begin{aligned}
\pi_{0} & =\left[1+\frac{\lambda}{\tilde{\lambda} \mu} \sum_{k=1}^{N-1}\left(\alpha^{*}\right)^{k-1}\right. \\
& \left.+\frac{1}{\theta}\left(\alpha^{*}\right)^{N-1} \sum_{k=N}^{\infty}(\alpha)^{k-N+1}\right]^{-1} .
\end{aligned}
$$

It is clear that if

$$
\sum_{k=N}^{\infty}(\alpha)^{k-N+1}<\infty
$$

there are

$$
\begin{aligned}
& 0<\pi_{0}<1, \\
& 0<\pi_{k}<1, \quad k \geq 1 .
\end{aligned}
$$

Then we obtain that the sufficient and necessary condition for the stability of the system is

$$
\alpha=\frac{\lambda \theta}{\mu(1-\lambda \theta)}<1 .
$$

If the above stability condition is fulfilled, it follows that

$$
\pi_{0}=\frac{\tilde{\lambda} \theta \mu\left(1-\alpha^{*}\right)(1-\alpha)}{\mu \theta(1-\lambda \theta)(1-\alpha)-\lambda \widetilde{\theta} \alpha\left(\alpha^{*}\right)^{N-1}} .
$$

Thus we get the following theorem.

Theorem 1. The sufficient and necessary condition for the stability of the system is

$$
\alpha=\frac{\lambda \theta}{\mu(1-\lambda \theta)}<1 .
$$

Remark 2. (1) When $\theta=0, \pi_{k}$ reduces to

$$
\pi_{k}= \begin{cases}\left(\frac{\lambda}{\tilde{\lambda} \mu}\right)^{k} \tilde{\mu}^{k-1} \pi_{0}^{*}, & 1 \leq k \leq N-1, \\ \frac{\lambda}{\mu}\left(\frac{\lambda \tilde{\mu}}{\tilde{\lambda} \mu}\right)^{N-1} \pi_{0}^{*}, & k=N,\end{cases}
$$

where $\pi_{0}^{*}=\left(\mu /(\mu-\lambda)-\left(\lambda^{2} / \mu(\mu-\lambda)\right)(\lambda \tilde{\mu} / \tilde{\lambda} \mu)^{N-1}\right)^{-1}$. It is the probability distribution of the number of customers in 
the standard $\mathrm{Geo} / \mathrm{Geo} / 1 / N$ queueing system in the late arrivals at observed epoch $\mathrm{m}^{+}$.

(2) When $\theta=1, \pi_{k}$ reduces to

$$
\pi_{k}=\left(\frac{\lambda}{\tilde{\lambda} \mu}\right)^{k} \pi_{0}, \quad k \geq 0
$$

where $\pi_{0}^{*}=1-\lambda / \widetilde{\lambda} \mu=1-\rho, \rho=\lambda / \widetilde{\lambda} \mu$. It is the probability distribution of the number of customers in the standard

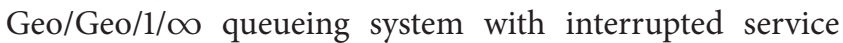
under the LCFS discipline in the late arrivals at observed epoch $\mathrm{m}^{+}$, and the sufficient and necessary condition for the stability of the system becomes $\lambda<\tilde{\lambda} \mu$.

\section{Performance Measures}

5.1. Loss Probability. In each moment the queue length of the system is either less than critical value $N$ or more than or equal to $N$. When the system state is more than or equal to $N$, the normal arrivals will be lost. So it is of interest to calculate the loss probability of the system in the stability. The loss probability $\pi_{l}$ is

$$
\begin{aligned}
\pi_{l} & =\sum_{k=N}^{\infty} \lambda \tilde{\theta} \pi_{k}=\sum_{k=N}^{\infty} \frac{\lambda \tilde{\theta}}{\theta}\left(\alpha^{*}\right)^{N-1}(\alpha)^{k-N+1} \pi_{0} \\
& =\frac{\lambda \tilde{\theta} \alpha\left(\alpha^{*}\right)^{N-1}}{\theta(1-\alpha)} \pi_{0} .
\end{aligned}
$$

5.2. Average Number of Customers in the System. Now we compute the expected values of the length of the queue $L$. From (18), we get

$$
\begin{aligned}
L & =\sum_{k=0}^{\infty} k \pi_{k}=\sum_{k=0}^{N-1} k \frac{\lambda}{\widetilde{\lambda} \mu}\left(\alpha^{*}\right)^{k-1} \pi_{0} \\
& +\sum_{k=N}^{\infty} k \frac{1}{\theta}\left(\alpha^{*}\right)^{N-1}(\alpha)^{k-N+1} \pi_{0} \\
& =\left(\frac{\lambda}{\tilde{\lambda} \mu}\left[\frac{1-\left(\alpha^{*}\right)^{N-1}}{\left(1-\alpha^{*}\right)^{2}}-\frac{(N-1)\left(\alpha^{*}\right)^{N-1}}{1-\alpha^{*}}\right]\right. \\
& \left.+\frac{\alpha\left(\alpha^{*}\right)^{N-1}[N-(N-1) \alpha]}{\theta(1-\alpha)^{2}}\right) \pi_{0} .
\end{aligned}
$$

5.3. The Virtual Busy Period. We will discuss a virtual busy period in the system which is different from the original system. The intention of the present paragraph is to facilitate discussion of the sojourn time of a customer. Only in this case, we suppose that the probability of an arrival is equal to $\lambda \theta$. Let $g_{k}, k \geq 0$, be the probability that the virtual busy period lasts $k$ slots. Then we have

$$
\begin{aligned}
g_{0}= & 0, \\
g_{k}= & (1-\lambda \theta)^{k} \tilde{\mu}^{k-1} \mu \\
& +\sum_{i=1}^{k}(1-\lambda \theta)^{i-1} \lambda \theta \sum_{j=i-1}^{\infty} \tilde{\mu}^{j} \mu \sum_{l=0}^{k-i} g_{l} g_{k-i-l},
\end{aligned}
$$

$$
k \geq 1 \text {. }
$$

We will use the generating function $g(x)=\sum_{k=0}^{\infty} x^{k} g_{k}$ to calculate it. Multiplying by $x^{k}$ in the above expression and summing over $k$. That has the following form:

$$
g(x)=\frac{\mu x(1-\lambda \theta)+\lambda \theta x g^{2}(x)}{1-\tilde{\mu} x(1-\lambda \theta)} .
$$

From expressions (31), we can show that $g(x)$ satisfies the quadratic equation

$$
f(g(x))=0,
$$

where

$$
\begin{aligned}
f(g)= & \lambda \theta x g^{2}(x)-[1-\tilde{\mu} x(1-\lambda \theta)] g(x) \\
& +\mu x(1-\lambda \theta) .
\end{aligned}
$$

Note that, for fixed $x_{0} \in(0,1)$, we get

$$
\begin{aligned}
\lambda \theta x_{0} & >0, \\
f(0) & =\mu x_{0}(1-\lambda \theta)>0, \\
f(1) & =\lambda \theta x_{0}-\left[1-\tilde{\mu} x_{0}(1-\lambda \theta)\right]+\mu x_{0}(1-\lambda \theta) \\
& =x_{0}-1<0 .
\end{aligned}
$$

Above relations show that, for any $x \in(0,1)$, the quadratic equation (32) has two solutions, $g(x)$ and $g^{*}(x)$, and satisfies the inequalities $0<g(x)<1<g^{*}(x)$ in which

$$
\begin{aligned}
& g(x) \\
& =\frac{1-\tilde{\mu} x(1-\lambda \theta)-\sqrt{[1-\tilde{\mu} x(1-\lambda \theta)]^{2}-4 \lambda \theta \mu x^{2}(1-\lambda \theta)}}{2 \lambda \theta x}, \\
& g^{*}(x) \\
& =\frac{1-\tilde{\mu} x(1-\lambda \theta)+\sqrt{[1-\tilde{\mu} x(1-\lambda \theta)]^{2}-4 \lambda \theta \mu x^{2}(1-\lambda \theta)}}{2 \lambda \theta x} .
\end{aligned}
$$

The probability generating function of the virtual busy period is $g(x)$, because $g^{*}(x)>1$.

It is not difficult to prove that expression $g(x)$ satisfies $g(1)=1$. Hence we can obtain the mean value of a virtual busy period as

$$
\bar{g}=g^{\prime}(1)=\frac{1}{\mu(1-\lambda \theta)(1-\alpha)} .
$$


5.4. The Actual Busy Period. In this paragraph, we will discuss the actual busy period of the system. An actual busy period $B$ is defined as the period from the epoch of the arrival of a customer who finds the system empty to that of the departure of a customer at which the system first becomes empty again. We will denote by $B_{k}, k \geq 0$, the probability that the actual busy period lasts $k$ slots. For giving its expression simply, we define the discrete $n$-fold convolution as follows:

$$
\begin{aligned}
& B_{k}^{1 *}=B_{k}, \\
& B_{k}^{2 *}=(B * B)_{k}=\sum_{i=0}^{k} P\{B=i\} P\{B=k-i\}, \\
& B_{k}^{n *}=\left(B^{(n-1) *} * B\right)_{k},
\end{aligned}
$$

$$
n \geq 1, k \geq 0 .
$$

Note that, in the condition that there is no arriving of the preferred customers in the first $i$ slots, the (conditional) probability that the normal customer arrives at the each slot $m, 1 \leq m \leq i$, is equal to $\lambda \widetilde{\theta} /(1-\lambda \theta)$. Thus, the probability, $B_{k}$, is given by

$$
\begin{aligned}
B_{0} & =0, \\
B_{k} & =\sum_{i=1}^{k}(1-\lambda \theta)^{i} \tilde{\mu}^{i-1} \mu \\
& \cdot \sum_{m=0}^{i} C_{i}^{m}\left(\frac{1-\lambda}{1-\lambda \theta}\right)^{i-m}\left(\frac{\lambda \tilde{\theta}}{1-\lambda \theta}\right)^{m} B_{k-i}^{\min \{m, N-1\} *} \\
& +\sum_{i=1}^{k}(1-\lambda \theta)^{i-1} \lambda \theta \sum_{j=i-1}^{\infty} \tilde{\mu}^{j} \mu \sum_{l=0}^{i-1} C_{i-1}^{l}\left(\frac{1-\lambda}{1-\lambda \theta}\right)^{i-1-l} \\
& \cdot\left(\frac{\lambda \tilde{\theta}}{1-\lambda \theta}\right)^{l} B_{k-i}^{\min \{l+2, N+1\} *}, \quad k \geq 1 .
\end{aligned}
$$

The first term in the right-hand side of (39) means that after a customer arrives (i.e., the beginning of busy period), there is no preferred arrival in the following $i$ slots except the normal arrivals, who could arrive or not at each slot before $i$ (the arriving occurs at the moment immediately before the slot boundary $i$ ), and in this situation only no more than $N-1$ customers could enter into the system, and the original customer finishes his service at the $i$ th slot (immediately after the $i$ th slot boundary $i$, to be more exact). Then the duration of the sum for serving $\min \{m, N-1\}$ customers lasts $k-i$ slots. The second term in the right-hand side can be explained similarly.

We define $B(x)=\sum_{k=0}^{\infty} x^{k} B_{k}$ as the probability generating function of busy period $B$. For getting it, we multiply by $x^{k}$ in (39) and sum over $k$. The result is

$$
\begin{aligned}
B(x) & =\frac{\tilde{\lambda} \mu x}{1-\tilde{\lambda} \tilde{\mu} x}+\frac{\lambda \tilde{\theta} \mu x B(x)}{(1-\tilde{\lambda} \tilde{\mu} x)^{2}}+\frac{(\lambda \tilde{\theta})^{2} \tilde{\mu} \mu x^{2} B^{2}(x)}{(1-\tilde{\lambda} \tilde{\mu} x)^{3}} \\
& +\frac{(\lambda \tilde{\theta})^{3}(\tilde{\mu})^{2} \mu x^{3} B^{3}(x)}{(1-\tilde{\lambda} \tilde{\mu} x)^{4}}+\cdots
\end{aligned}
$$

$$
\begin{aligned}
& +\frac{(\lambda \tilde{\theta})^{N-2}(\tilde{\mu})^{N-3} \mu x^{N-2} B^{N-2}(x)}{(1-\tilde{\lambda} \tilde{\mu} x)^{N-1}} \\
& +\sum_{m=N-1}^{\infty} \frac{(\lambda \tilde{\theta})^{m}(\tilde{\mu})^{m-1} \mu x^{m} B^{N-1}(x)}{(1-\tilde{\lambda} \tilde{\mu} x)^{m+1}}+\frac{\lambda \theta x B^{2}(x)}{1-\tilde{\lambda} \tilde{\mu} x} \\
& +\frac{(1-\tilde{\lambda} \tilde{\mu} x)^{2}+\frac{\lambda \theta(\lambda \tilde{\theta})^{2}(\tilde{\mu})^{2} x^{3} B^{4}(x)}{(1-\tilde{\lambda} \tilde{\mu} x)^{3}}}{\lambda \theta(\lambda \tilde{\theta}))^{N-2}(\tilde{\mu})^{2} B^{3}(x) x^{N-1} B^{N}(x)} \\
& +\cdots+\frac{(1-\tilde{\lambda} \tilde{\mu} x)^{N-1}}{(1 \theta)}
\end{aligned}
$$$$
+\sum_{m=N-1}^{\infty} \frac{\lambda \theta(\lambda \tilde{\theta})^{m}(\tilde{\mu})^{m} x^{m+1} B^{N+1}(x)}{(1-\tilde{\lambda} \tilde{\mu} x)^{m+1}}=\frac{\tilde{\lambda} \mu x}{1-\tilde{\lambda} \tilde{\mu} x}
$$$$
+\sum_{m=1}^{N-2} \frac{(\lambda \tilde{\theta})^{m}(\tilde{\mu})^{m-1} \mu x^{m} B^{m}(x)}{(1-\tilde{\lambda} \tilde{\mu} x)^{m+1}}
$$$$
+\sum_{m=N-1}^{\infty} \frac{(\lambda \widetilde{\theta})^{m}(\tilde{\mu})^{m-1} \mu x^{m} B^{N-1}(x)}{(1-\tilde{\lambda} \tilde{\mu} x)^{m+1}}
$$$$
+\sum_{m=0}^{N-2} \frac{\lambda \theta(\lambda \tilde{\theta})^{m}(\tilde{\mu})^{m} x^{m+1} B^{m+2}(x)}{(1-\tilde{\lambda} \tilde{\mu} x)^{m+1}}
$$$$
+\sum_{m=N-1}^{\infty} \frac{\lambda \theta(\lambda \tilde{\theta})^{m}(\tilde{\mu})^{m} x^{m+1} B^{N+1}(x)}{(1-\tilde{\lambda} \tilde{\mu} x)^{m+1}}=\frac{\tilde{\lambda} \mu x}{1-\tilde{\lambda} \tilde{\mu} x}
$$$$
+\frac{\lambda \tilde{\theta} \mu x B(x)}{(1-\tilde{\lambda} \tilde{\mu} x)(1-\tilde{\lambda} \tilde{\mu} x-\lambda \tilde{\theta} \tilde{\mu} x B(x))}[1
$$$$
\left.-\left(\frac{\lambda \tilde{\theta} \tilde{\mu} x B(x)}{1-\tilde{\lambda} \tilde{\mu} x}\right)^{N-2}\right]
$$$$
+\frac{(\lambda \widetilde{\theta})^{N-1} \tilde{\mu}^{N-2} \mu x^{N-1} B^{N-1}(x)}{(1-\tilde{\lambda} \tilde{\mu} x)^{N-1}(1-\tilde{\lambda} \tilde{\mu} x-\lambda \tilde{\theta} \tilde{\mu} x)}
$$$$
+\frac{\lambda \theta x B^{2}(x)}{1-\tilde{\lambda} \tilde{\mu} x-\lambda \widetilde{\theta} \tilde{\mu} x B(x)}[1
$$$$
\left.-\left(\frac{\lambda \tilde{\theta} \tilde{\mu} x B(x)}{1-\tilde{\lambda} \tilde{\mu} x}\right)^{N-1}\right]
$$$$
+\frac{(\lambda \tilde{\theta})^{N-1} \lambda \theta \widetilde{\mu}^{N-1} x^{N} B^{N+1}(x)}{(1-\tilde{\lambda} \tilde{\mu} x)^{N-1}(1-\tilde{\lambda} \tilde{\mu} x-\lambda \tilde{\theta} \tilde{\mu} x)} .
$$ 
Here, we only list the computing process of the fourth term in (40) (i.e., for $m=3$ ). Rewrite (40) as the following form:

$$
B_{k}=B_{1, k}+B_{2, k}, \quad k \geq 1,
$$

in which

$$
\begin{aligned}
& B_{2, k}=\sum_{i=1}^{k}(1-\lambda \theta)^{i-1} \lambda \theta \sum_{j=i-1}^{\infty} \tilde{\mu}^{j} \mu \sum_{l=0}^{i-1} C_{i-1}^{l}\left(\frac{1-\lambda}{1-\lambda \theta}\right)^{i-1-l} \\
& \cdot\left(\frac{\lambda \tilde{\theta}}{1-\lambda \theta}\right)^{l} B_{k-i}^{\min \{l+2, N+1\} *}, \\
& B_{1, k}=\sum_{i=1}^{k}(1-\lambda \theta)^{i} \tilde{\mu}^{i-1} \mu \sum_{m=0}^{i} C_{i}^{m}\left(\frac{1-\lambda}{1-\lambda \theta}\right)^{i-m} \\
& \cdot\left(\frac{\lambda \tilde{\theta}}{1-\lambda \theta}\right)^{m} B_{k-i}^{\min \{m, N-1\} *}=\sum_{i=1}^{k}(1-\lambda \theta)^{i} \\
& \cdot \tilde{\mu}^{i-1} \mu\left[C_{i}^{0}\left(\frac{1-\lambda}{1-\lambda \theta}\right)^{i}\right. \\
&+C_{i}^{1}\left(\frac{1-\lambda}{1-\lambda \theta}\right)^{i-1}\left(\frac{\lambda \tilde{\theta}}{1-\lambda \theta}\right)^{1} B_{k-i}^{1 *}+\cdots \\
&\left.+C_{i}^{i}\left(\frac{\lambda \tilde{\theta}}{1-\lambda \theta}\right)^{i} B_{k-i}^{\min \{i, N-1\} *}\right] .
\end{aligned}
$$

Multiplying by $x^{k}$ and summing over $k$, the fourth term in the above expression of the right-hand side becomes

$$
\begin{gathered}
\sum_{k=1}^{\infty} x^{k}\left\{\sum _ { i = 1 } ^ { k } ( 1 - \lambda \theta ) ^ { i } \tilde { \mu } ^ { i - 1 } \mu \left[C_{i}^{3}\left(\frac{1-\lambda}{1-\lambda \theta}\right)^{i-3}\right.\right. \\
\left.\left.\cdot\left(\frac{\lambda \widetilde{\theta}}{1-\lambda \theta}\right)^{3} \sum_{j_{1}=1}^{k-i} \sum_{j_{2}=1}^{k-i-j_{1}} B_{j_{1}} B_{j_{2}} B_{k-i-j_{1}-j_{2}}\right]\right\} \\
=\sum_{i=1}^{\infty} x^{i} \widetilde{\mu}^{i-1} \mu C_{i}^{3}(1-\lambda)^{i-3}(\lambda \widetilde{\theta})^{3} B^{3}(x)
\end{gathered}
$$

(exchanging the order of summing)

$$
\begin{aligned}
& =(\lambda \widetilde{\theta})^{3} \widetilde{\mu}^{2} \mu x^{3} B^{3}(x) \sum_{i=1}^{\infty} C_{i}^{3}(1-\lambda)^{i-3} \tilde{\mu}^{i-3} x^{i-3} \\
& =(\lambda \widetilde{\theta})^{3} \tilde{\mu}^{2} \mu x^{3} B^{3}(x) \frac{1}{3 !} \sum_{i=1}^{\infty} i(i-1)(i-2)(\widetilde{\lambda} \tilde{\mu} x)^{i-3} \\
& =(\lambda \widetilde{\theta})^{3} \tilde{\mu}^{2} \mu x^{3} B^{3}(x) \frac{1}{3 !} \sum_{i=0}^{\infty} i(i-1)(i-2)(y)^{i-3}
\end{aligned}
$$

(where $y=\tilde{\lambda} \widetilde{\mu} x$ )

$$
\begin{aligned}
= & (\lambda \widetilde{\theta})^{3} \widetilde{\mu}^{2} \mu x^{3} B^{3}(x) \frac{1}{3 !} \frac{d^{3}}{d y}\left[\sum_{i=0}^{\infty} y^{i}\right]=(\lambda \widetilde{\theta})^{3} \\
& \cdot \tilde{\mu}^{2} \mu x^{3} B^{3}(x) \frac{1}{3 !} \frac{d^{3}}{d y}\left(\frac{1}{1-y}\right)=(\lambda \widetilde{\theta})^{3} \\
& \cdot \tilde{\mu}^{2} \mu x^{3} B^{3}(x) \frac{1}{3 !} \frac{3 !}{(1-y)^{4}}=\frac{(\lambda \widetilde{\theta})^{3} \tilde{\mu}^{2} \mu x^{3} B^{3}(x)}{(1-\tilde{\lambda} \tilde{\mu} x)^{4}} .
\end{aligned}
$$

Now we show that the solution of (40) is of existence and uniqueness within the unit circle of the complex plane by Rouchés theorem, and it must be $B(x)$.

Define $z=B(x)$ and the right-hand side of (40) as $g(z)$; that is,

$$
\begin{aligned}
& g(z)=\frac{\tilde{\lambda} \mu x}{1-\tilde{\lambda} \tilde{\mu} x}+\frac{\lambda \tilde{\theta} \mu x z}{(1-\tilde{\lambda} \tilde{\mu} x)^{2}}+\frac{(\lambda \tilde{\theta})^{2} \tilde{\mu} \mu x^{2} z^{2}}{(1-\tilde{\lambda} \tilde{\mu} x)^{3}} \\
& +\frac{(\lambda \tilde{\theta})^{3}(\tilde{\mu})^{2} \mu x^{3} z^{3}}{(1-\tilde{\lambda} \tilde{\mu} x)^{4}}+\cdots \\
& +\frac{(\lambda \widetilde{\theta})^{N-2}(\widetilde{\mu})^{N-3} \mu x^{N-2} z^{N-2}}{(1-\tilde{\lambda} \tilde{\mu} x)^{N-1}} \\
& +\sum_{m=N-1}^{\infty} \frac{(\lambda \widetilde{\theta})^{m}(\widetilde{\mu})^{m-1} \mu x^{m} z^{N-1}}{(1-\tilde{\lambda} \tilde{\mu} x)^{m+1}}+\frac{\lambda \theta x z^{2}}{1-\tilde{\lambda} \widetilde{\mu} x} \\
& +\frac{\lambda \theta(\lambda \tilde{\theta}) \tilde{\mu} x^{2} z^{3}}{(1-\tilde{\lambda} \widetilde{\mu} x)^{2}}+\frac{\lambda \theta(\lambda \widetilde{\theta})^{2}(\widetilde{\mu})^{2} x^{3} z^{4}}{(1-\tilde{\lambda} \tilde{\mu} x)^{3}}+\cdots \\
& +\frac{\lambda \theta(\lambda \widetilde{\theta})^{N-2}(\widetilde{\mu})^{N-2} x^{N-1} z^{N}}{(1-\tilde{\lambda} \widetilde{\mu} x)^{N-1}} \\
& +\sum_{m=N-1}^{\infty} \frac{\lambda \theta(\lambda \tilde{\theta})^{m}(\tilde{\mu})^{m} x^{m+1} z^{N+1}}{(1-\tilde{\lambda} \tilde{\mu} x)^{m+1}},
\end{aligned}
$$

which is a complex analytic function of variable $z$.

Thus, (40) could be rewritten as

$$
z-g(z)=0 .
$$

Obviously, $B(x), 0 \leq x \leq 1$, is a root of the above equation. For any $x \in[0,1)$ and $|z|=1$, there is

$$
\begin{aligned}
|g(z)| \leq & \frac{\tilde{\lambda} \mu x}{1-\tilde{\lambda} \tilde{\mu} x}+\frac{\lambda \tilde{\theta} \mu x}{(1-\tilde{\lambda} \tilde{\mu} x)^{2}}+\frac{(\lambda \tilde{\theta})^{2} \tilde{\mu} \mu x^{2}}{(1-\tilde{\lambda} \tilde{\mu} x)^{3}} \\
& +\frac{(\lambda \tilde{\theta})^{3}(\tilde{\mu})^{2} \mu x^{3}}{(1-\tilde{\lambda} \tilde{\mu} x)^{4}}+\cdots
\end{aligned}
$$




$$
\begin{aligned}
& +\frac{(\lambda \tilde{\theta})^{N-2}(\widetilde{\mu})^{N-3} \mu x^{N-2}}{(1-\tilde{\lambda} \tilde{\mu} x)^{N-1}} \\
& +\sum_{m=N-1}^{\infty} \frac{(\lambda \widetilde{\theta})^{m}(\tilde{\mu})^{m-1} \mu x^{m}}{(1-\tilde{\lambda} \tilde{\mu} x)^{m+1}}+\frac{\lambda \theta x}{1-\tilde{\lambda} \widetilde{\mu} x} \\
& +\frac{\lambda \theta(\lambda \widetilde{\theta}) \tilde{\mu} x^{2}}{(1-\tilde{\lambda} \widetilde{\mu} x)^{2}}+\frac{\lambda \theta(\lambda \widetilde{\theta})^{2}(\widetilde{\mu})^{2} x^{3}}{(1-\tilde{\lambda} \widetilde{\mu} x)^{3}}+\cdots \\
& +\frac{\lambda \theta(\lambda \widetilde{\theta})^{N-2}(\widetilde{\mu})^{N-2} x^{N-1}}{(1-\widetilde{\lambda} \widetilde{\mu} x)^{N-1}} \\
& +\sum_{m=N-1}^{\infty} \frac{\lambda \theta(\lambda \widetilde{\theta})^{m}(\widetilde{\mu})^{m} x^{m+1}}{(1-\tilde{\lambda} \tilde{\mu} x)^{m+1}} \\
& =\frac{\tilde{\lambda} \mu x}{1-\tilde{\lambda} \tilde{\mu} x}+\frac{\lambda \tilde{\theta} \mu x}{(1-\tilde{\lambda} \tilde{\mu} x)(1-\tilde{\lambda} \tilde{\mu} x-\lambda \tilde{\theta} \tilde{\mu} x)} \\
& +\frac{\lambda \theta x}{1-\widetilde{\lambda} \widetilde{\mu} x-\lambda \tilde{\theta} \widetilde{\mu} x}=\frac{x-\tilde{\lambda} \tilde{\mu} x-\lambda \tilde{\theta} \tilde{\mu} x}{1-\tilde{\lambda} \widetilde{\mu} x-\lambda \widetilde{\theta} \tilde{\mu} x}<1 \\
& =|z| .
\end{aligned}
$$

Hence, within the unit circle by Rouchés theorem, the number of roots of $z-g(z)$ is the same as that of $z$, which only has one root obviously. Also for any probability generating function $B(x)=\sum_{k=0}^{\infty} x^{k} B_{k}$, there is $|B(x)|<1$ for any $x \in$ $[0,1)$, which implies that $z=B(x), 0 \leq x<1$, is the unique solution of $z-g(z)=0($ or $(40))$ within the unit circle $|z|=1$.

Further, for $x=1$ we have $g(1)=1$ and $z-\left.g(z)\right|_{z=1}=$ $1-g(1)=0$. So root $z=B(x)$ satisfies $B(1)=1$ as well.

Thus, the following result is proven.

Theorem 3. The solution of (40) exists and there is just one probability generating function $B(x), 0 \leq x \leq 1$, satisfying it.

The mean of busy period is

$$
\begin{aligned}
E[B] & =B^{\prime}(1) \\
& =\frac{(1-\tilde{\lambda} \widetilde{\mu})^{N-1}}{(\lambda \widetilde{\theta})^{N} \widetilde{\mu}^{N-1}+(\mu-\lambda-\lambda \theta \mu)(1-\tilde{\lambda} \widetilde{\mu})^{N-1}} .
\end{aligned}
$$

Remark 4. (1) For $\theta=0, N \rightarrow \infty$, (40) becomes

$$
B(x)=\frac{\mu x[\widetilde{\lambda}+\lambda B(x)]}{1-\widetilde{\mu} x[\widetilde{\lambda}+\lambda B(x)]},
$$

which is the same as the probability generating function of the busy period in the standard Geo/Geo/1/o queueing system in the late arrivals [14, pp 203].
(2) For $\theta=0, N=2,(40)$ becomes

$$
B(x)=\frac{\tilde{\lambda} \mu x(1-\tilde{\mu} x)}{1-\tilde{\lambda} \tilde{\mu} x-\tilde{\mu} x-\lambda \mu x+\widetilde{\lambda} \widetilde{\mu}^{2} x^{2}},
$$

which is the same as the probability generating function of the busy period in the standard $\mathrm{Geo} / \mathrm{Geo} / 1 / 2$ queueing system in the late arrivals [15, pp 236].

(3) For $N \rightarrow \infty$, (40) becomes

$$
B(x)=\frac{\mu x[\widetilde{\lambda}+\lambda \widetilde{\theta} B(x)]+\lambda \theta x B^{2}(x)}{1-\tilde{\mu} x(\widetilde{\lambda}+\lambda \widetilde{\theta} B(x))} .
$$

We can simply get the probability generating function

$$
B(x)=\frac{1-\tilde{\lambda} \tilde{\mu} x-\lambda \widetilde{\theta} \mu x}{2(\lambda \theta+\lambda \widetilde{\theta} \tilde{\mu}) x}-m(x),
$$

where

$$
\begin{aligned}
& m(x) \\
& \quad=\left[(1-\tilde{\lambda} \tilde{\mu} x-\lambda \tilde{\theta} \mu x)^{2}-4 \widetilde{\lambda} \mu(\lambda \theta+\lambda \tilde{\theta} \widetilde{\mu}) x^{2}\right]^{1 / 2} \\
& \cdot(2 \lambda \theta x+2 \lambda \tilde{\theta} \tilde{\mu} x)^{-1} .
\end{aligned}
$$

5.5. Sojourn Time of a Customer in a Server. The intention of this section is to study the distribution of the time that a customer spends in the server in the steady state. If a new preferred customer arrives to the system, the service will be interrupted. Hence the sojourn time of a customer in the server is the elapsed service times plus a whole service time again after interruptions. We let $h_{k}, k \geq 0$, be the probability that the sojourn time of a customer in the server lasts $k$ slots. Then the distribution $\left\{h_{k} ; k \geq 0\right\}$ is given by

$$
\begin{aligned}
& h_{0}=0, \\
& h_{k}=(1-\lambda \theta)^{k} \tilde{\mu}^{k-1} \mu+\sum_{i=1}^{k}(1-\lambda \theta)^{i-1} \lambda \theta \sum_{j=i-1}^{\infty} \widetilde{\mu}^{j} \mu h_{k-i},
\end{aligned}
$$

$$
k \geq 1 \text {. }
$$

We define $h(x)=\sum_{k=0}^{\infty} x^{k} h_{k}$ as the probability generating function of sojourn time of a customer in the server. For getting it, we multiply by $x^{k}$ in (54) and sum over $k$. The result is

$$
h(x)=\frac{\mu x(1-\lambda \theta)}{1-\widetilde{\mu} x(1-\lambda \theta)}+\frac{\lambda \theta x h(x)}{1-\widetilde{\mu} x(1-\lambda \theta)}
$$

or

$$
\begin{aligned}
h(x) & =\frac{\mu x(1-\lambda \theta)}{1-\tilde{\mu} x-\lambda \theta \mu x}=\frac{\mu(1-\lambda \theta) x}{1-[1-\mu(1-\lambda \theta)] x} \\
& =\mu(1-\lambda \theta) \sum_{i=0}^{\infty}[1-\mu(1-\lambda \theta)]^{k} x^{k+1} .
\end{aligned}
$$


Thus, we obtain the probability distribution of $\left\{h_{k} ; k \geq 0\right\}$ as follows:

$$
\begin{aligned}
& h_{0}=0, \\
& h_{k}=\mu(1-\lambda \theta)[1-\mu(1-\lambda \theta)]^{k-1}, \quad k \geq 1 .
\end{aligned}
$$

Theorem 5. The distribution of the time that a customer spends in the server in the system follows a geometric distribution with parameter $\mu(1-\lambda \theta)$.

The mean time of a customer in the server is

$$
\bar{h}=\frac{1}{\mu(1-\lambda \theta)} .
$$

5.6. The Virtual Sojourn Time of a Customer in the System. In this section we attempt to present the distribution of the virtual sojourn time that a customer spends in the system. A virtual sojourn time of a customer in the system is defined as the period of time that a customer spends in the system from the beginning of its service till the moment of its departure. About this system, we will reckon the possible interruptions time on the sojourn time. We denote by $s_{k}, k \geq 0$, the probability that the virtual sojourn time of a customer in the system lasts $k$ slots. It is given by

$$
\begin{aligned}
s_{0}= & 0, \\
s_{k}= & (1-\lambda \theta)^{k} \tilde{\mu}^{k-1} \mu \\
& +\sum_{i=1}^{k}(1-\lambda \theta)^{i-1} \lambda \theta \sum_{m=i-1}^{\infty} \tilde{\mu}^{m} \mu \sum_{j=0}^{k-i} g_{j} s_{k-i-j},
\end{aligned}
$$

$$
k \geq 1 \text {. }
$$

We also use the generating function $s(x)=\sum_{k=0}^{\infty} x^{k} s_{k}$ to calculate it. That has the following form:

$$
s(x)=\frac{\mu x(1-\lambda \theta)+\lambda \theta x g(x) s(x)}{1-\tilde{\mu} x(1-\lambda \theta)},
$$

or

$$
s(x)=\frac{\mu x(1-\lambda \theta)}{1-\tilde{\mu} x(1-\lambda \theta)-\lambda \theta x g(x)} .
$$

The mean time of a customer in the system is given by

$$
\bar{s}=s^{\prime}(1)=\frac{1}{\mu(1-\lambda \theta)(1-\alpha)} .
$$

5.7. The Actual Sojourn Time of a Customer in the System. Let $M_{k}, k \geq 0$, be that the probability of the actual sojourn time of the new arrivals in the system lasts $k$ slots. An actual sojourn time of a customer in the system is defined as the period of time that a customer spends in the system from the beginning of the entrance into the system till the moment of its departure. Hence, the actual sojourn time of the new customer that arrives in the system is the sum of two parttimes: the time from the beginning of the entrance into the system to the contacting server and the virtual sojourn time of a customer in the system. It is given by

$$
\begin{aligned}
& M_{0}= 0 \\
& M_{k}=\frac{\left[s_{k} \pi_{0}+\sum_{m=1}^{\infty} s_{k} \theta \pi_{m}+\sum_{m=1}^{N-1}\left(g^{m *} * s\right)_{k} \tilde{\theta} \pi_{m}\right]}{\left(1-\pi_{l} / \lambda\right)}, \\
& k \geq 1 .
\end{aligned}
$$

We also use the generating function $M(x)=\sum_{k=0}^{\infty} x^{k} M_{k}$ to calculate the mean value of the sojourn time of a customer in the system. Note (63) that has following form:

$$
\begin{aligned}
& M(x)=\frac{s(x)}{1-\pi_{l} / \lambda}\left[\theta+\widetilde{\theta} \pi_{0}\right. \\
& \left.+\frac{\lambda \tilde{\theta} \pi_{0}\left[1-\left(\alpha^{*} g(x)\right)^{N-1}\right] g(x)}{\tilde{\lambda} \mu\left(1-\alpha^{*} g(x)\right)}\right] .
\end{aligned}
$$

The mean sojourn time of a customer in the system is given by

$$
\begin{aligned}
& M^{\prime}(1)=\frac{1}{\mu(1-\lambda \theta)(1-\alpha)}[1 \\
& \left.+\frac{\lambda \tilde{\theta} \pi_{0}\left(1+(\tilde{\lambda} \mu-1) \alpha^{*}-N\left(\alpha^{*}\right)^{N-1}+(N-\tilde{\lambda} \mu)\left(\alpha^{*}\right)^{N}\right)}{\tilde{\lambda} \mu\left(1-\pi_{l} / \lambda\right)\left(1-\alpha^{*}\right)^{2}}\right] \\
& \quad=\frac{1}{\mu(1-\lambda \theta)(1-\alpha)}\{1 \\
& \left.+\frac{\lambda \tilde{\theta} \pi_{0}}{\tilde{\lambda} \mu\left(1-\pi_{l} / \lambda\right)}\left[\frac{1-\left(\alpha^{*}\right)^{N-1}}{\left(1-\alpha^{*}\right)^{2}}-\frac{(N-1)\left(\alpha^{*}\right)^{N-1}}{1-\alpha^{*}}\right]\right\} .
\end{aligned}
$$

5.8. Waiting Time. Let $W_{k}, k \geq 0$, be that the probability of the waiting time of the new arrivals in the system lasts $k$ slots. A waiting time of a customer in the system is defined as the period of time that a customer spends in the system from the beginning of the entrance into the system till the moment of its departure minus the time spent in the sever. Hence, the waiting time of the new customer that arrives in the system is the time of the actual sojourn time of the customer in the system minus the time spent in the server. It is given by

$$
\sum_{i=0}^{k}\left(W_{i} * h_{k-i}\right)=M_{k}, \quad k \geq 0 .
$$

So the generating function of the waiting time of the new arrivals in the system is

$$
M(x)=W(x) h(x)
$$

or

$$
W(x)=\frac{M(x)}{h(x)}
$$




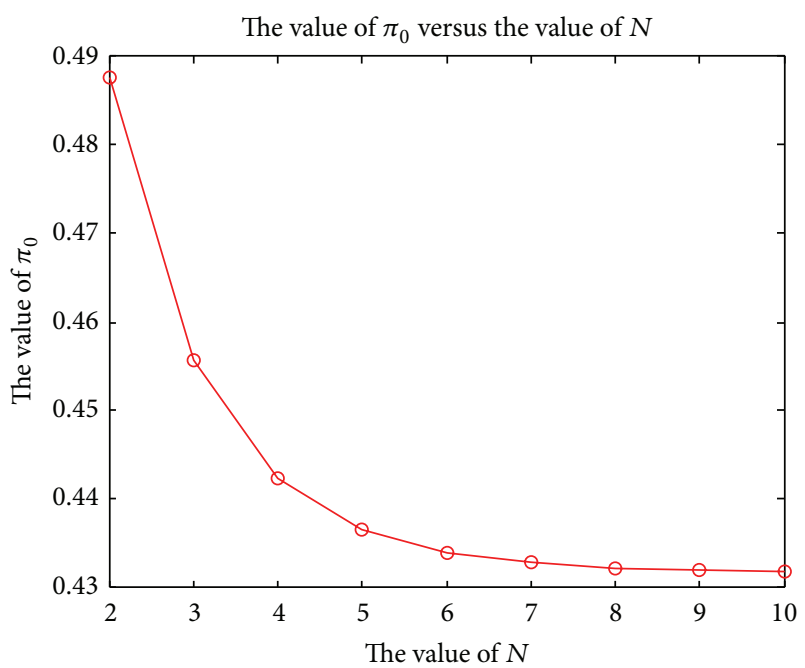

FIGURE 1: The free probability versus threshold $N$ where $\lambda=0.3$, $\theta=0.4, \mu=0.6$.

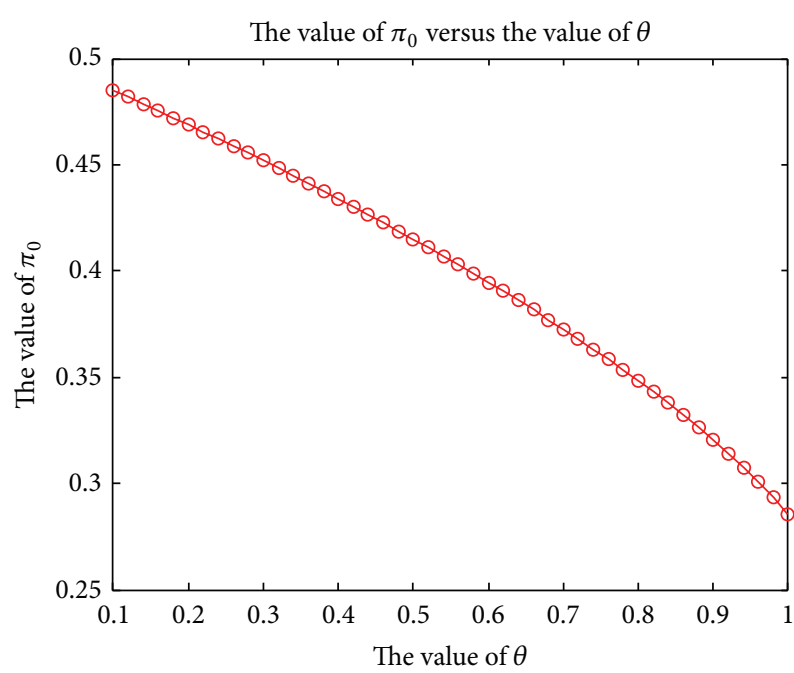

FIgURE 2: The free probability versus $\theta$ where $N=6, \lambda=0.3, \mu=$ 0.6 .

The mean waiting time of a customer in the system is given by

$$
\begin{gathered}
W^{\prime}(1)=\left.\frac{M^{\prime}(x) h(x)-M(x) h^{\prime}(x)}{h^{2}(x)}\right|_{x=1}=M^{\prime}(1) \\
-h^{\prime}(1)=\frac{1}{\mu(1-\lambda \theta)(1-\alpha)}\{1 \\
+\frac{\lambda \tilde{\theta} \pi_{0}}{\widetilde{\lambda} \mu\left(1-\pi_{l} / \lambda\right)}\left[\frac{1-\left(\alpha^{*}\right)^{N-1}}{\left(1-\alpha^{*}\right)^{2}}\right. \\
\left.\left.-\frac{(N-1)\left(\alpha^{*}\right)^{N-1}}{1-\alpha^{*}}\right]\right\}-\frac{1}{\mu(1-\lambda \theta)} .
\end{gathered}
$$

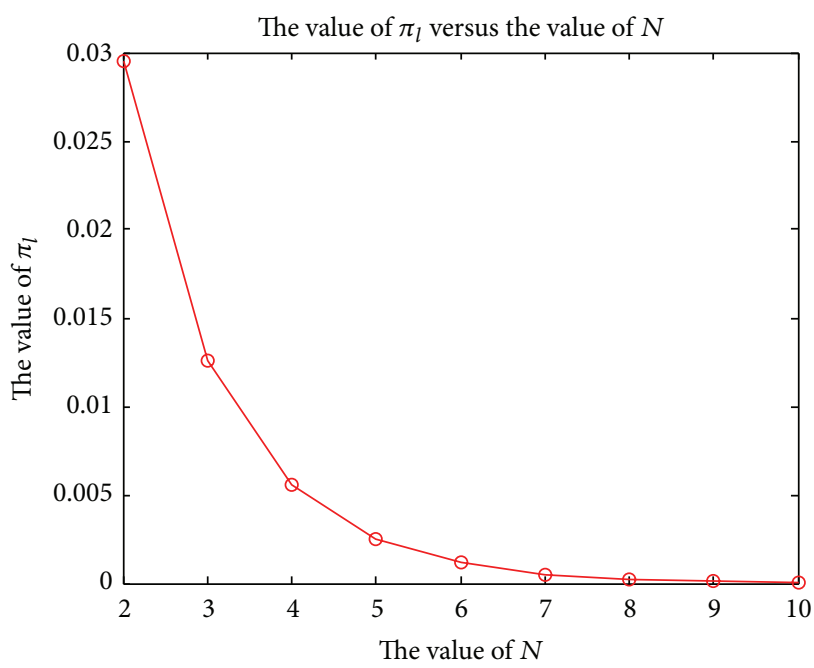

FIgURE 3: The loss probability versus threshold $N$ where $\lambda=0.3$, $\theta=0.4, \mu=0.6$.

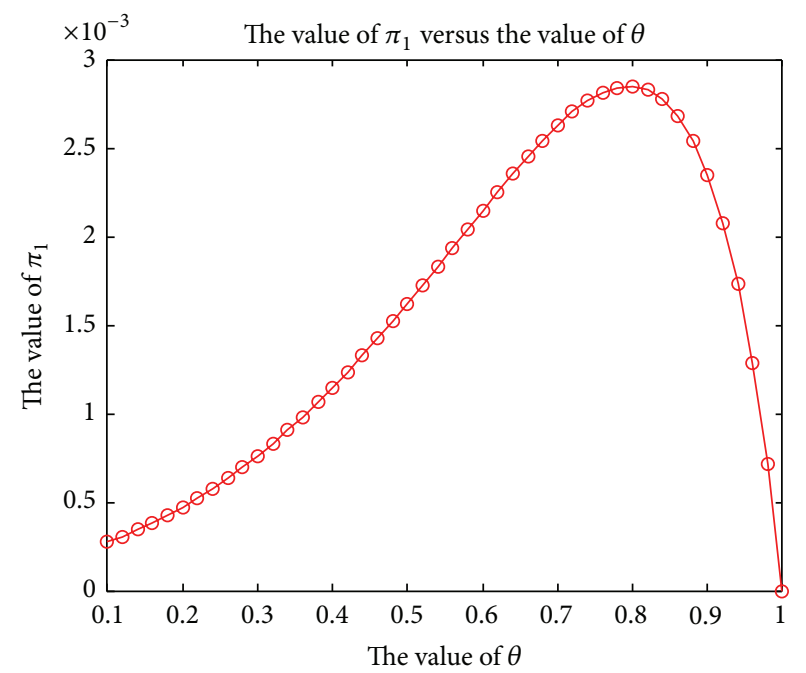

Figure 4: The loss probability versus $\theta$ where $N=6, \lambda=0.3, \mu=$ 0.6 .

\section{Numerical Results}

In this section, we provide some numerical examples to show the effect of the main parameters $\theta$ and $N$ on the performance characteristics. We consider six performance measures: the free probability $\pi_{0}$, the loss probability $\pi_{l}$, the mean system size $L$, the mean busy period $B^{\prime}(1)$, the mean sojourn time in the system $M^{\prime}(1)$, and the mean waiting time $W^{\prime}(1)$. Of course in all the below examples, the parametric values are chosen so as to satisfy the stability condition. We derive the results by using two cases as follows.

Case 1. $\lambda=0.3, \theta=0.4, \mu=0.6$ and varying the values of $N$ from 5 to 40 .

Case 2. $N=20, \lambda=0.3, \mu=0.6$ and varying the values of $\theta$ from 0.1 to 1 . 


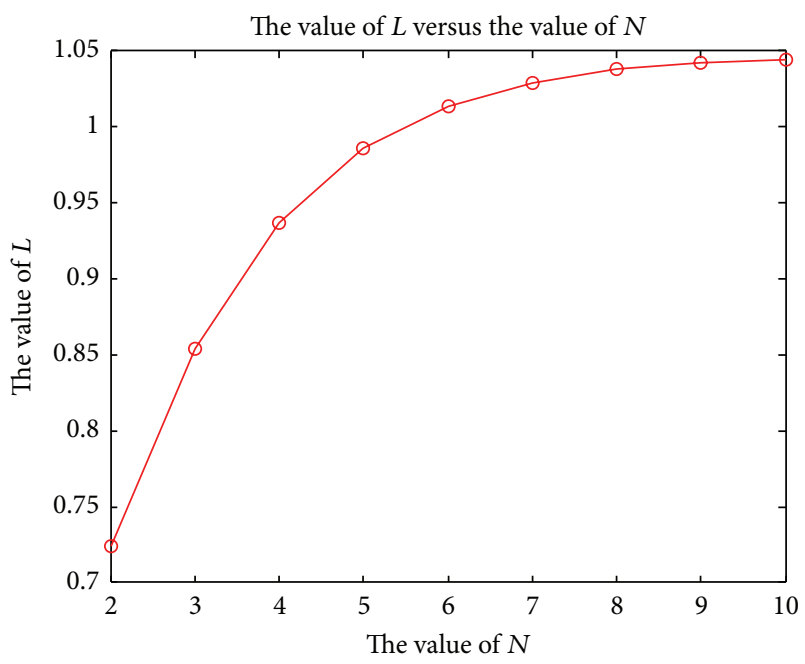

FIgURE 5: The mean system size versus threshold $N$ where $\lambda=0.3$, $\theta=0.4, \mu=0.6$.

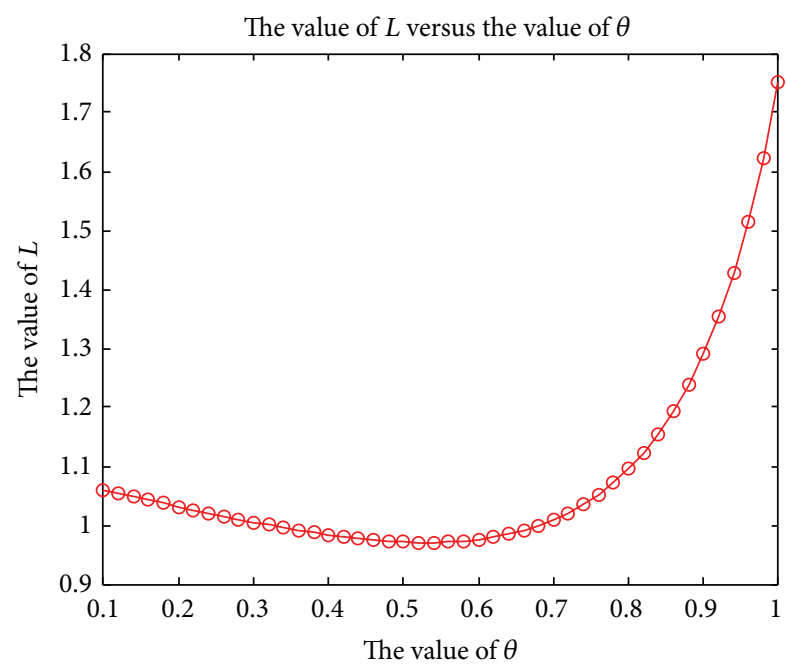

Figure 6: The mean system size versus $\theta$ where $N=6, \lambda=0.3$, $\mu=0.6$.

Case 1 reveals a situation in which the threshold $N$ changes from 2 to 10 by one. As expected, every performance measure is insensitive to the change in $N$ because we consider the queue with partial buffer control (the partial customers are restricted). In Figures 1 and 3, the probabilities that the system is empty and system is lost are plotted against parameter $N$. The curves which in decreasing order correspond to bigger threshold $N$ show, as intuition tells us, that, for increasing values of the parameter $N$, the probabilities of an empty system and the ignored customers decrease. Just the opposite, the remaining four indicators (the mean system size, the mean busy period, the mean sojourn time in the system, and the mean waiting time) increase more rapidly.

In Case 2, parameter $\theta$ changes from 0.1 to 1 by 0.02 . Increase in $\theta$ means increase in the preferred arrivals and at the same time decrease in the normal arrivals. Figure 2 show that as $\theta$ increases the free probability decreases very rapidly.

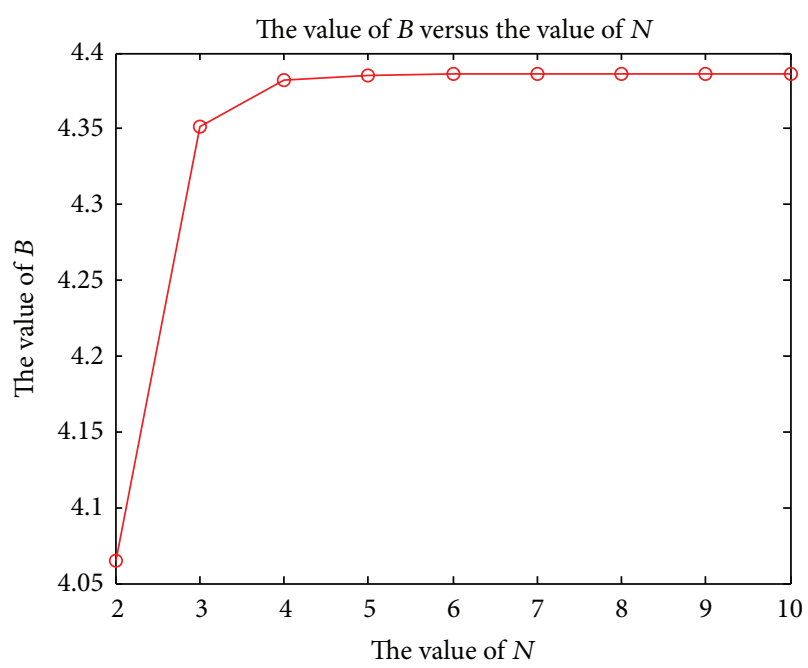

Figure 7: The mean of busy period versus threshold $N$ where $\lambda=$ $0.3, \theta=0.4, \mu=0.6$.

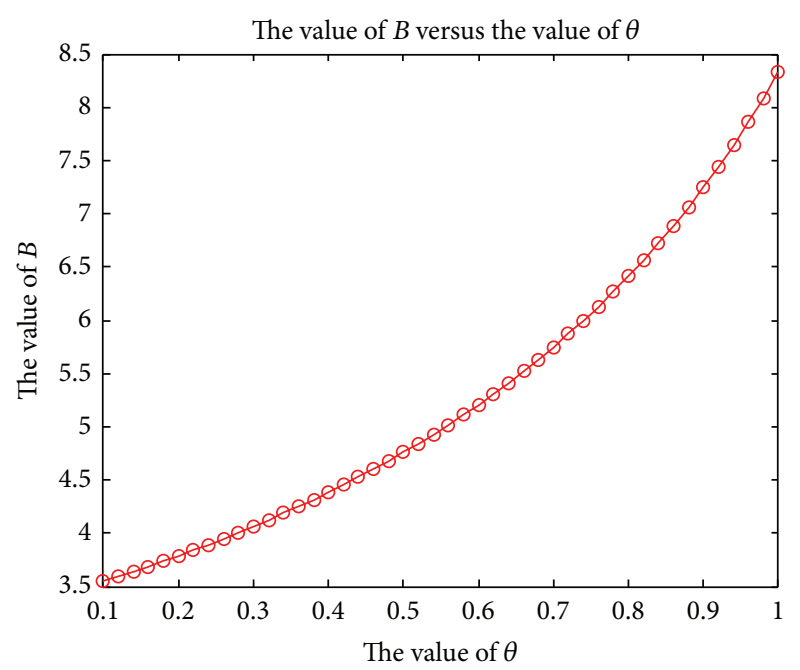

Figure 8: The mean of busy period versus $\theta$ where $N=6, \lambda=0.3$, $\mu=0.6$.

The three indicators (the mean busy period, the mean sojourn time in the system, and the mean waiting time) increase more rapidly. Particularly curious are the indicators of the loss probability and mean system size. Although the arrival rate of the normal customers is decreased, as it can be seen from Figure 4 , the curve of the loss probability $\pi_{l}$ increases monotonously from $\theta=0.1$ to $\theta=0.8$. The mean system size versus threshold $N$ is shown in Figure 5. Then it declines sharply from $\theta=0.8$ to $\theta=1$. This is caused by the increased preferred customers making the service occupied which leads to increase in the initial graph. When the preferred customers continue to increase until $\theta=1$, it is obvious that the number of the normal customers reduces in the system. Thereby the loss probability decreases until 0 . In Figure 6, the mean system size and the loss probability are inverse trends which is also very obvious fact. The mean of busy period versus threshold $N$ is shown in Figure 7. The mean of busy period 


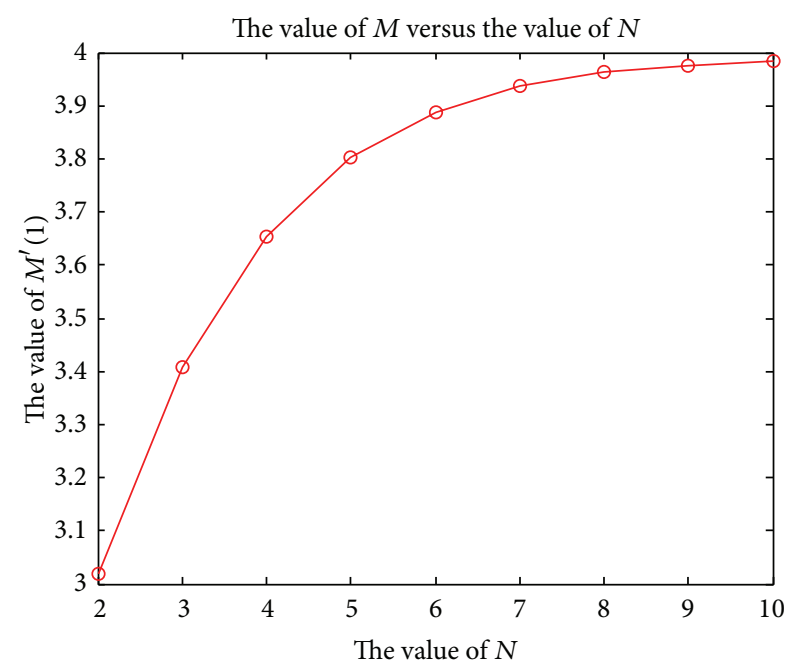

FIgURE 9: The mean sojourn time in the system versus $N$ where $\lambda=$ $0.3, \theta=0.4, \mu=0.6$.

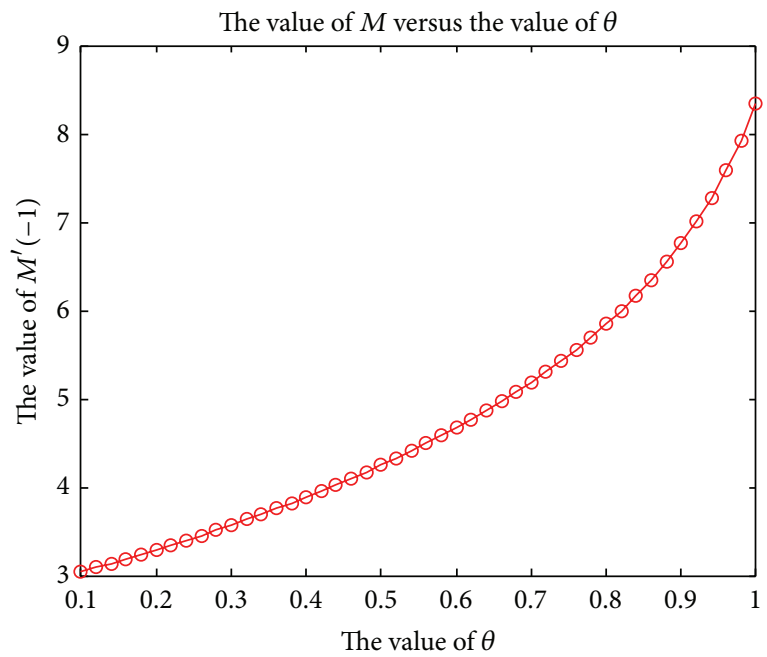

FIGURE 10: The mean sojourn time in the system versus $\theta$ where $N=$ $6, \lambda=0.3, \mu=0.6$.

versus $\theta$ is shown in Figure 8. The mean sojourn time in the system versus $N$ is shown in Figure 9. The mean sojourn time in the system versus $\theta$ is shown in Figure 10. The mean waiting time versus $N$ is shown in Figure 11. The mean waiting time versus $\theta$ is shown in Figure 12 .

\section{Conclusion}

As everyone knows, the analysis of a queue system with partial buffer sharing is much more difficult than that with infinite capacity, especially under the priority mechanism. In this paper, we study a discrete-time $\mathrm{Geo} / \mathrm{Geo} / 1$ queue with preferred customers and partial buffer sharing and carry out an extensive investigation. A sufficient and necessary condition for the stability of the system (Theorem 1) and the stationary distribution of queue length (18) are obtained.

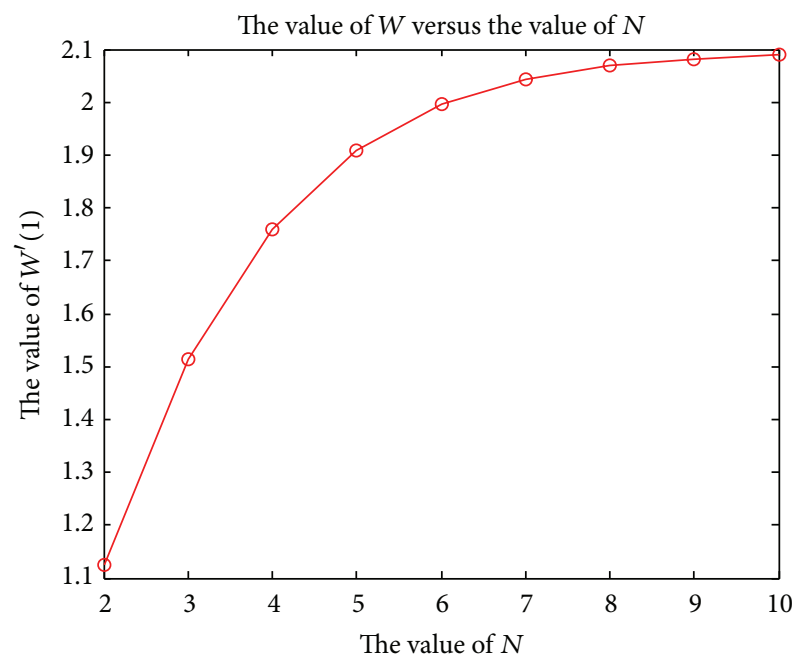

FIGURE 11: The mean waiting time versus $N$ where $\lambda=0.3, \theta=0.4$, $\mu=0.6$.

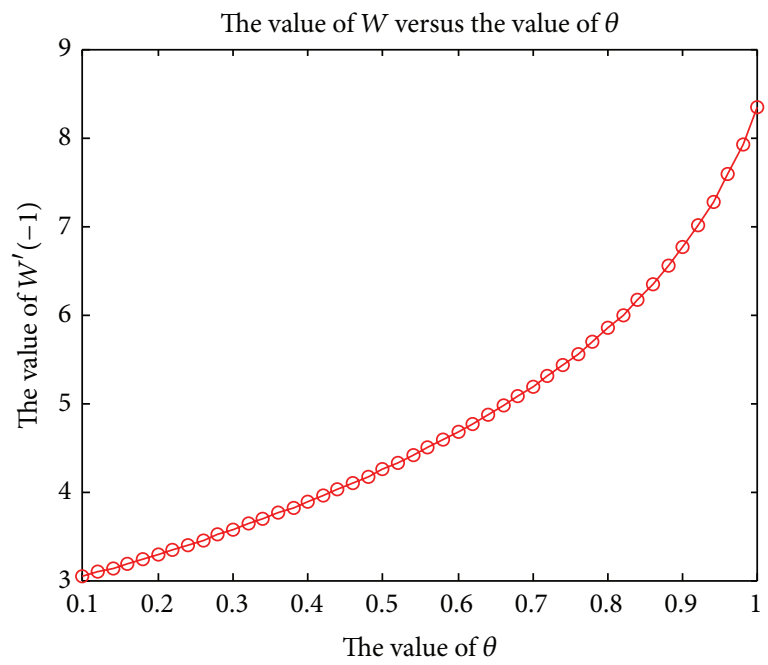

FIGURE 12: The mean waiting time versus $\theta$ where $N=6, \lambda=0.3$, $\mu=0.6$.

By using a novel method, we get the probability generating function of busy period of the system in the steady state and prove its existence and uniqueness (Theorem 3) and discuss some boundary value problems. Finally, by calculating the virtual busy period and virtual sojourn time of a customer in the system, we obtain the distribution of the time that a customer spends in the server, the actual sojourn time of a customer in the system, and waiting time. Particularly, we derive the distribution of the time that a customer spends in the server, which follows a geometric distribution (Theorem 5).

\section{Conflict of Interests}

The authors declare that there is no conflict of interests regarding the publication of this paper. 


\section{References}

[1] H. Takagi, Queueing Analysis: A Foundation of Performance Evaluation in Discrete-Time Systems, vol. 3, North-Holland, Amsterdam, The Netherlands, 1993.

[2] T. Meisling, "Discrete-time queuing theory," Operations Research, vol. 6, pp. 96-105, 1958.

[3] P. P. Bocharov, C. D’Apice, A. V. Pechinkin, and S. Salerno, Queueing Theory, VSP, Utrecht, The Netherlands, 2004.

[4] H. Daduna, Queueing Networks with Discrete-Time Scale, Springer, Berlin, Germany, 2001.

[5] I. Atencia and P. Moreno, "A single-server G-queue in discretetime with geometrical arrival and service process," Performance Evaluation, vol. 59, no. 1, pp. 85-97, 2005.

[6] U. C. Gupta and V. Goswami, "Performance analysis of finite buffer discrete-time queue with bulk service," Computers \& Operations Research, vol. 29, no. 10, pp. 1331-1341, 2002.

[7] Z. G. Zhang and N. Tian, "Discrete time Geo/G/1 queue with multiple adaptive vacations," Queueing Systems, vol. 38, no. 4, pp. 419-429, 2001.

[8] F. Jolai, S. M. Asadzadeh, and M. R. Taghizadeh, "A preemptive discrete-time priority buffer system with partial buffer sharing," Applied Mathematical Modelling, vol. 34, no. 8, pp. 2148-2165, 2010.

[9] S. Ndreca and B. Scoppola, "Discrete time GI/Geom/1 queueing system with priority," European Journal of Operational Research, vol. 189, no. 3, pp. 1403-1408, 2008.

[10] J. Walraevens, B. Steyaert, and H. Bruneel, "Performance analysis of a GI/Geo/1 buffer with a preemptive resume priority scheduling discipline," European Journal of Operational Research, vol. 157, no. 1, pp. 130-151, 2004.

[11] H. W. Lee, S. H. Lee, S. H. Yoon, B. Y. Ahn, and N. I. Park, "A recursive method for Bernoulli arrival queues and its application to partial buffer sharing in ATM," Computers \& Operations Research, vol. 26, no. 6, pp. 559-581, 1999.

[12] I. Atencia and A. V. Pechinkin, "A discrete-time queueing system with optional LCFS discipline," Annals of Operations Research, vol. 202, pp. 3-17, 2013.

[13] J. B. Wu, J. X. Wang, and Z. M. Liu, "A discrete-time Geo/G/1 retrial queue with preferred and impatient customers," Applied Mathematical Modelling, vol. 37, no. 4, pp. 2552-2561, 2013.

[14] O. W. W. Yang, "Performance comparison of some discrete service time systems," Performance Evaluation, vol. 23, no. 3, pp. 261-284, 1995.

[15] R. Sun and J. Li, Foundation of Queueing Theory, China Science Press, 2002. 


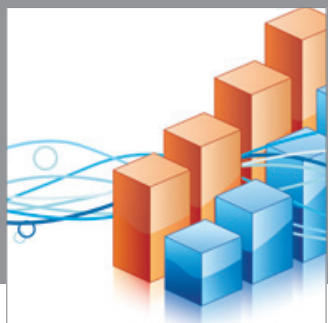

Advances in

Operations Research

mansans

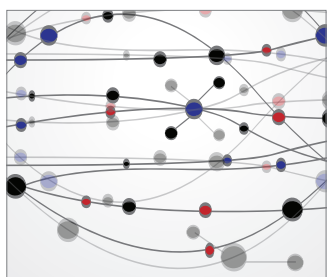

The Scientific World Journal
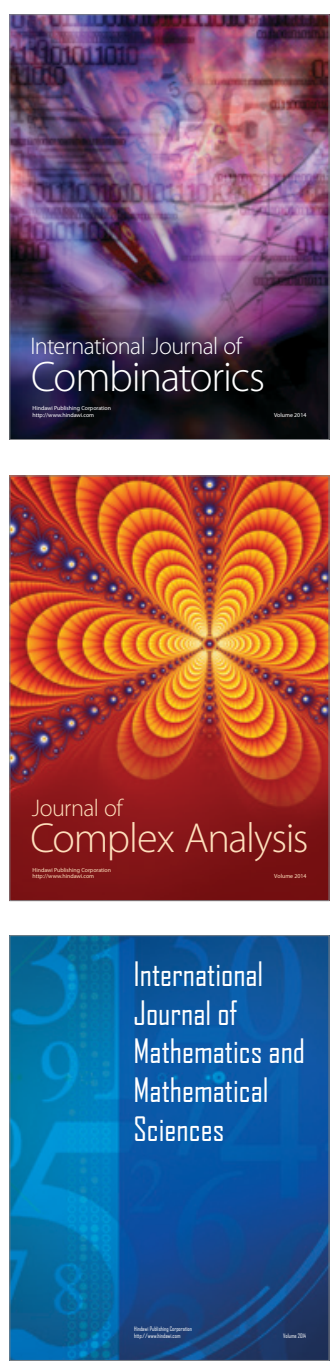
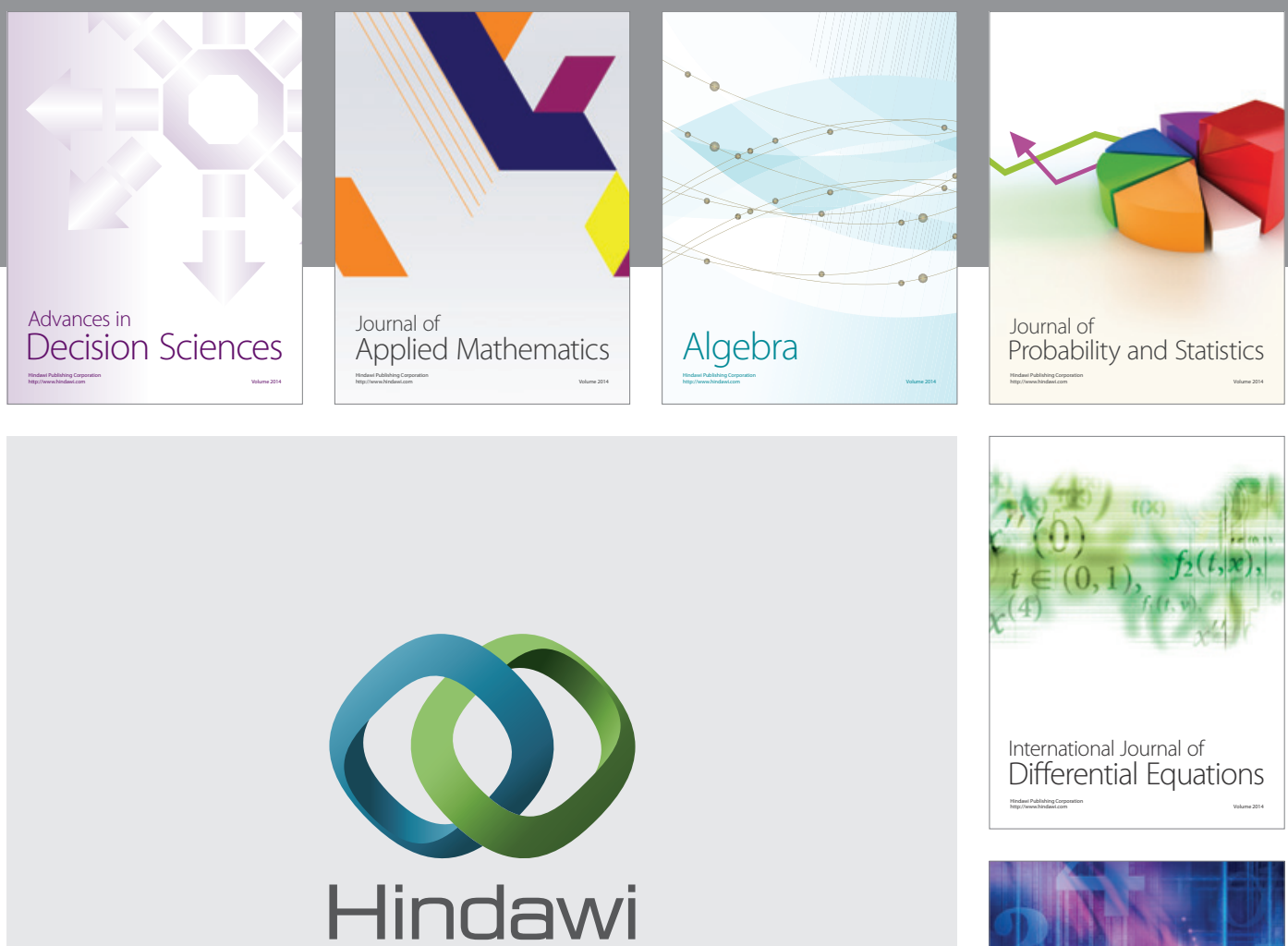

Submit your manuscripts at http://www.hindawi.com
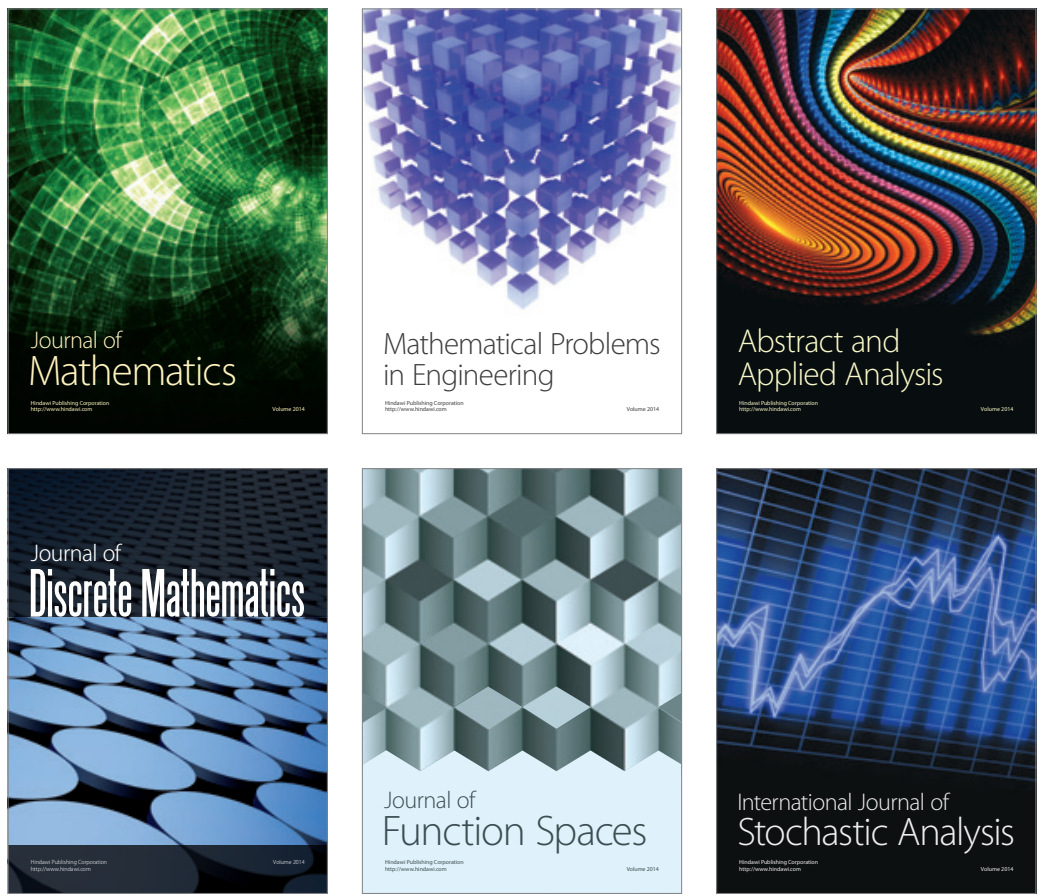

Journal of

Function Spaces

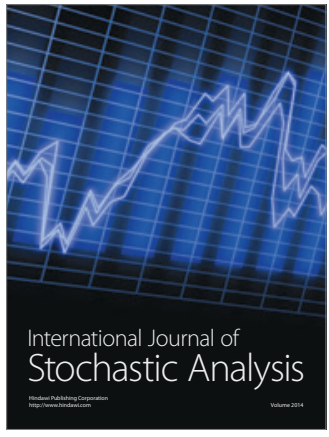

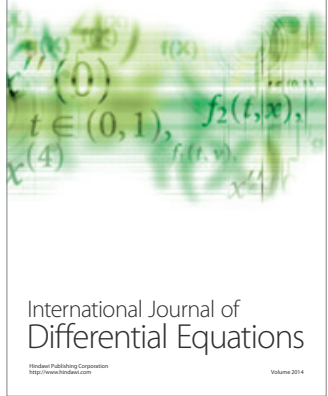
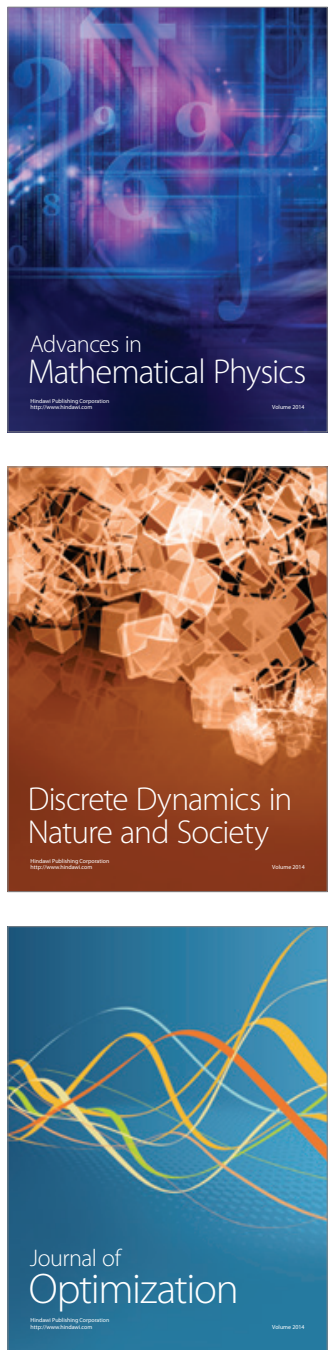